\title{
Predicción de los grandes síndromes obstétricos
}

\section{Prediction of major obstetric syndromes}

\section{Juan P. Kusanovic 1,2,3,4*}

${ }^{1}$ División de Obstetricia y Ginecología, Escuela de Medicina, Pontificia Universidad Católica de Chile, Santiago, Chile; ${ }^{2}$ Unidad de Alto Riesgo Obstétrico, Centro de Investigación e Innovación en Medicina Materno-Fetal (CIMAF), Hospital Dr. Sótero del Río, Santiago, Chile; ${ }^{3}$ Perinatology Research Branch, NICHD/NIH/DHHS, Detroit, USA; ${ }^{4}$ Departmento de Obstetricia y Gynecología, Wayne State University, Detroit, USA

«It is tough to make predictions, especially about the futurew."

"(Antiguo proverbio danés)".

Hace 122 años, John William Ballantyne manifestó que el manejo del trabajo de parto, el parto y el puerperio había tenido grandes avances, pero que diversas patologías, como el aborto, la infertilidad, el embarazo gemelar, el nacimiento de fetos malformados y la muerte fetal, entre otras, no habían recibido la misma atención' ${ }^{1}$. En aquellos años, las mujeres embarazadas que necesitaban hospitalización eran ingresadas bajo el cuidado de médicos generales sin experiencia particular en obstetricia. Para mejorar el diagnóstico y el tratamiento antenatales de las complicaciones del embarazo, Ballantyne publicó en 1901 Plea for a ProMaternity Hospital ${ }^{2}$. En esta publicación defendía el desarrollo de un hospital de maternidad como una necesidad fundamental para que los obstetras pudieran estudiar tanto el embarazo normal como el patológico. Propuso, además, que los objetivos del cuidado prenatal deberían ser prevenir las convulsiones, tratar las enfermedades maternas e identificar las malformaciones congénitas antes del parto («to prevent fits, treat maternal diseases, and to identify monsters antenata$\| y »)^{3}$. Incluso, a pesar de encontrarse en el epílogo del siglo XIX, sus propuestas fueron extremadamente visionarias, como el desarrollo de tratamientos antenatales ("the possibility of antenatal therapeutics»), el tratamiento posnatal de patologías antenatales («postnatal treatment of antenatal and intranatal morbid states»), el manejo de condiciones durante el trabajo de parto y el parto («intranatal therapeutics»), e incluso la posibilidad de desarrollar tratamientos intrauterinos («ante-natal treatment: intrauterine») ${ }^{4}$.

"It is, of course, everywhere admitted that much may be done after labour, even in labour, to cure or at least to ameliorate morbid states arising during antenatal life; but this is not usually regarded as antenatal therapeutics in the strict sense of the term. It may be claimed, however, that it is an integral part of the subject, and I look upon the claim as one that must be yielded.»

Más de 100 años después, la medicina materno-fetal es capaz de diagnosticar síndromes clínicos, pero no los mecanismos fisiopatológicos específicos que dan origen y caracterizan a cada una de las patologías más importantes del embarazo. Un denominador común para la mayoría de las condiciones que se asocian a mortalidad y morbilidad perinatal son el trabajo de parto y el parto prematuro, rotura prematura de membranas, la preeclampsia, la restricción del crecimiento intrauterino y la muerte fetal ${ }^{5}$. En primer lugar, el término «trabajo de parto prematuro» no nos orienta a si esta patología está causada por una infección intraamniótica o sistémica, o si se debe a disfunción cervical, sobredistensión uterina, estrés materno, lesiones vasculares placentarias, alergia u otros mecanismos de
Disponible en internet: 02-08-2021 Rev Chil Obstet Ginecol. 2021;86(3):255-257 www. rechog.com 0048-766X / ๑ 2021 Sociedad Chilena de Obstetricia y Ginecología. Publicado por Permanyer. Éste es un artículo open access bajo la licencia CC BY-NC-ND (https://creativecommons.org/licenses/by-nc-nd/4.0/). 
enfermedad. En segundo lugar, muchos síndromes obstétricos se manifiestan clínicamente con respuestas adaptativas asociadas a ciertas condiciones fisiopatológicas ${ }^{3}$. Un ejemplo tradicional es la preeclampsia, la cual es una respuesta a alteraciones tempranas en la resistencia vascular a nivel útero-placentario, siendo el aumento de la presión arterial una manifestación y no la causa de la preeclampsia 6 . De esta forma, considerar que una única prueba diagnóstica detectará cada una de estas complicaciones del embarazo significa ignorar la naturaleza «sindrómica» de todas estas patologías. Asimismo, esto explica por qué diversas intervenciones, como la progesterona, el cerclaje o los tocolíticos, no son eficaces en el tratamiento del parto prematuro asociado a una infección intraamniótica, o por qué el régimen hiposódico y el uso de antihipertensivos no mejoran el resultado perinatal en las pacientes con preeclampsia. Es interesante que la mayoría de los síndromes obstétricos tienen una larga fase subclínica y sus expresiones clínicas son manifestaciones tardías de los procesos fisiopatológicos que los ocasionan ${ }^{5}$. Por ejemplo, la infección intraamniótica ${ }^{7,8}$ y el acortamiento cervical ${ }^{9-11}$ pueden ser asintomáticos y asociarse con un resultado adverso perinatal semanas después; igualmente, las alteraciones del Doppler de las arterias uterinas ${ }^{12-14}$ y los cambios en las concentraciones de factores angiogénicos y antiangiogénicos ${ }^{15-17}$ preceden en semanas al desarrollo de un cuadro de preeclampsia o de restricción del crecimiento fetal.

En este número de nuestra revista, dos estudios se centran en la predicción de la preeclampsia y el parto prematuro. López-Jurado, et al. evaluaron el rendimiento diagnóstico de un nomograma diseñado por Alegría y Gonzales $^{18}$ para la predicción de la preeclampsia, el cual combina características clínicas y ecográficas, incluyendo paridad, edad materna, antecedente de preeclampsia o familiares directas con este antecedente, presencia de comorbilidad, índice de masa corporal, presión arterial media, proteinuria de 24 horas e índice de pulsatilidad promedio de las arterias uterinas, lo cual permite asignar un puntaje a cada una de estas variables. Cuanto más alto sea el puntaje asignado por el nomograma, mayor es el riesgo de desarrollar preeclampsia (190 puntos para bajo riesgo, 230 puntos para riesgo intermedio y 320 puntos para alto riesgo). El estudio incluyó 513 pacientes atendidas en el servicio de ginecología y obstetricia del Hospital Nacional Dos de Mayo, en Perú. Los autores encontraron que un valor de corte de 187 puntos tiene el mejor rendimiento diagnóstico para la predicción de preeclampsia, con una sensibilidad del $81 \%$ y una especificidad del $86 \%$.

Por otra parte, Reyna-Villasmil, et al. compararon la medición de la longitud cervical con el índice de consistencia cervical en la predicción de parto prematuro inminente (definido como parto dentro de 7 días) en pacientes sintomáticas. Este fue un estudio prospectivo que incluyó 657 pacientes con diagnóstico de amenaza de parto prematuro entre las 24 y 35 semanas de gestación, y que fueron atendidas en el Hospital Central Dr. Urquinaona, en Venezuela. Las pacientes que tuvieron un parto prematuro dentro de 7 días presentaron valores significativamente más bajos del índice de consistencia y longitud cervicales, comparadas con las controles. Los autores hallaron que el área bajo la curva de la longitud cervical fue significativamente mayor que la del índice de consistencia, concluyendo que este índice no es superior a la longitud cervical en la predicción del parto prematuro dentro de 7 días en pacientes sintomáticas.

Las múltiples etiologías de cada uno de estos síndromes hacen que sea poco probable que una sola prueba identifique todos o la mayoría de los casos. La baja prevalencia de estas enfermedades hace que un número muy importante de mujeres con embarazos normales deban ser sometidas a pruebas de cribado. Sin embargo, incluso con una baja tasa de falsos positivos y una alta sensibilidad, el valor predictivo positivo de una prueba diagnóstica seguirá siendo muy bajo, ya que la mayoría de las condiciones que intentamos predecir son infrecuentes ${ }^{3}$. Por lo tanto, la definición de los procesos patológicos, la identificación de biomarcadores específicos y la implementación de intervenciones terapéuticas en el primer trimestre son un enorme desafío para la medicina materno-fetal del nuevo milenio.

\section{Bibliografía}

1. Reiss HE. Historical insights: John William Ballantyne 1861-1923. Hum Reprod Update. 1999;5:386-9.

2. Ballantyne JW. A plea for a pro-maternity hospital. Br Med J. 1901:1:813-4

3. Romero R. Prenatal medicine: the child is the father of the man. 1996. J Matern Fetal Neonatal Med. 2009;22:636-9.

4. Ballantyne JW. On Antenatal Therapeutics: Being the Concluding Lecture of a Series of Six on Antenatal Pathology and Teratology Delivered in the University of Edinburgh in February and March, 1899. Br Med J. 1899;1:889-93.

5. Di Renzo GC. The great obstetrical syndromes. J Matern Fetal Neonatal Med. 2009;22:633-5.

6. Romero R, Lockwood C, Oyarzun E, Hobbins JC. Toxemia: new concepts in an old disease. Semin Perinatol. 1988;12:302-23.

7. Gray DJ, Robinson HB, Malone J, Thomson RB Jr. Adverse outcome in pregnancy following amniotic fluid isolation of Ureaplasma urealyticum. Prenat Diagn. 1992;12:111-7.

8. Horowitz S, Mazor M, Romero R, Horowitz J, Glezerman M. Infection of the amniotic cavity with Ureaplasma urealyticum in the mid trimester of pregnancy. J Reprod Med. 1995;40:375-9. 
9. Hassan SS, Romero R, Berry SM, Dang K, Blackwell SC, Treadwell MC, et al. Patients with an ultrasonographic cervical length $<$ or $=15 \mathrm{~mm}$ have nearly a $50 \%$ risk of early spontaneous preterm delivery. Am J Obstet Gynecol. 2000:182:1458-67.

10. To MS, Skentou C, Liao AW, Cacho A, Nicolaides KH. Cervical length and funneling at 23 weeks of gestation in the prediction of spontaneous early preterm delivery. Ultrasound Obstet Gynecol. 2001:18:200-3.

11. Parra-Cordero M, Sepúlveda-Martínez A, Rencoret G, Valdés E, Pedraza D, Muñoz $\mathrm{H}$. Is there a role for cervical assessment and uterine artery Doppler in the first trimester of pregnancy as a screening test for spontaneous preterm delivery? Ultrasound Obstet Gynecol. 2014:43:291-6.

12. Velauthar L, Plana MN, Kalidindi M, Zamora J, Thilaganathan B, Illanes SE, et al. First-trimester uterine artery Doppler and adverse pregnancy outcome: a meta-analysis involving 55,974 women. Ultrasound Obstet Gynecol. 2014;43:500-7.

13. Sepúlveda-Martínez A, Rencoret G, Silva MC, Ahumada P, Pedraza D, Muñoz $\mathrm{H}$, et al. First trimester screening for preterm and term pre-eclampsia by maternal characteristics and biophysical markers in a low-risk population. J Obstet Gynaecol Res. 2019;45:104-12.
14. Mönckeberg M, Arias V, Fuenzalida R, Álvarez S, Toro V, Calvo A, et al. Diagnostic performance of first trimester screening of preeclampsia based on uterine artery pulsatility index and maternal risk factors in routine clinical use. Diagnostics (Basel). 2020:10:182.

15. Crispi F, Llurba E, Domínguez C, Martín-Gallán P, Cabero L, Gratacós E. Predictive value of angiogenic factors and uterine artery Doppler for early- versus late-onset pre-eclampsia and intrauterine growth restriction. Ultrasound Obstet Gynecol. 2008:31:303-9.

16. Sepúlveda-Martínez A, Garrido M, Caamano E, Vega M, Romero C, Parra-Cordero M. Maternal plasma nerve growth factor at the $11+0$ $13+6$ weeks' scan as a potential angiogenic marker of preeclampsia: a pilot study. Fetal Diagn Ther. 2017;41:202-8.

17. Nzelu D, Biris D, Karampitsakos T, Nicolaides KK, Kametas NA. First trimester serum angiogenic and anti-angiogenic factors in women with chronic hypertension for the prediction of preeclampsia. Am J Obstet Gynecol. 2020;222:374.e1-9.

18. Alegría-Guerrero R, Gonzales-Medina C. Evaluación diagnóstica de un nomograma de predicción de preeclampsia. Rev Peru Investig Materno Perinat. 2019;7:29-47. 